\title{
Clinical And Hematological Profile Of Febrile Neutropenia In Pediatric Patients Who Suffered From Malignancy At Dr. Soetomo General Academic Hospital Surabaya
}

\author{
Anggia Gracia Marlina Situmorang ${ }^{1}$, Puspa Wardhani ${ }^{2}$, I Dewa Gede Ugrasena ${ }^{3}$ \\ ${ }^{1}$ Email: anggia.gracia.marlina-2018@fk.unair.ac.id \\ ${ }^{1}$ Medical Program, Faculty of Medicine, Universitas Airlangga, Surabaya, 60132, Indonesia \\ ${ }^{2}$ Department of Clinical Pathology, Dr. Soetomo General Academic Hospital, Universitas Airlangga, Surabaya, 60132, Indonesia \\ ${ }^{3}$ Department of Pediatrics, Dr. Soetomo General Academic Hospital, Universitas Airlangga, Surabaya, 60132, Indonesia
}

\begin{abstract}
Background: Febrile neutropenia is a common complication found in the practice of hematology-oncology caused by malignancy and immune-stimulating cytotoxic therapy. The appearance of febrile neutropenia in patients with malignancy often has a negative impact on the patient's prognosis. There has been no research on febrile neutropenia, especially those using data from Dr. Soetomo General Academic Hospital Surabaya until now. This study aims to determine febrile neutropenia's clinical and hematological profile in pediatric patients who suffered from malignancy at Dr. Soetomo General Academic Hospital Surabaya. Methods: A retrospective study using the medical records of febrile neutropenic patients with malignancy at the Division of Hematology-Oncology, Department of Pediatrics, Dr. Soetomo General Academic Hospital Surabaya from January 2018 to December 2019. The descriptive analysis in this study describes the clinical and hematological profile of febrile neutropenia in pediatric patients who suffered from malignancy, including underlying clinical diagnosis, clinical symptoms, clinical signs, and hematological examination results. Results: Thirty pediatric patients with febrile neutropenia suffered from malignancy. From the data obtained, the most common clinical diagnosis underlying the incidence of febrile neutropenia was acute lymphoblastic leukemia (73\%). The most common clinical symptom experienced by patients was fever (100\%). The most common clinical sign experienced by patients was anemia $(67 \%)$. The most common hematological examination results were neutropenia $(100 \%)$ with absolute neutrophil count $<500$ cells $/ \mu \mathrm{L}$. Conclusion: Acute lymphoblastic leukemia dominates the clinical diagnosis underlying the development of febrile neutropenia in pediatric patients with malignancy. The most common clinical and hematological profiles were fever, anemia, and neutropenia.
\end{abstract}

Keywords: Febrile Neutropenia; Clinical Profile; Hematological Profile

\section{Introduction}

Febrile neutropenia is a common complication found in hematology-oncology practices. In Indonesia, based on studies conducted on pediatric patients with malignancies treated within one year from April 1, 2015, to April 30, 2016, at the Ministry of Child Health, Dr. Cipto Mangunkusumo Hospital Jakarta, there was a $100 \%$ neutropenia fever incidence with a variation of at least one and a maximum of 3 episodes for each patient [1]. Cases of neutropenia fever often occur due to malignancy and cytotoxic therapy that can change the clinical and hematological profile. The appearance of febrile neutropenia in patients with malignancies is often bad for the patient's prognosis [2]. 
Febrile neutropenia can have an impact on patients. Consequences of febrile neutropenia can include reduction and delay of chemotherapy doses so that adjustments are needed to the therapy received by the patient [3]. In addition, to restore the neutrophils count to $>500$ cells $/ \mu \mathrm{L}$, patients need to get additional treatment in the form of broad-spectrum antibiotics for a long time. Prolonged use of antibiotics can lead to the emergence of antibiotic-resistant bacteria. In a retrospective and prospective study in Japan on 126 pediatric patients suffering from malignancy, antibiotic cycling methods were used in several classes of antibiotics to reduce the onset of resistance [4]. Then, the economic burden will be more pronounced, especially in patients at high risk of febrile neutropenia. In a retrospective study through medical records data on 104,315 pediatric patients suffering from malignancy in 2007-2014 in the United States, it was seen that hospitalization costs increased from year to year with the highest middle value of $\$ 11,202$ achieved in 2014 [5].

Febrile neutropenia is a fever associated with a low neutrophil count that indicate secondary conditions due to malignancy (cancer) and drugs that stimulate immunity. Specifically, neutropenia can occur as a result of bone marrow injury caused by cancer, chemotherapy, radiation therapy, other underlying disease processes, and combinations. Results of inpatient and outpatient studies showed a 16.8\% risk of developing febrile neutropenia during chemotherapy [6]. Fever occurs due to the release of endogenous cytokines by epithelial cells. Febrile neutropenia is characterized by a single oral temperature greater than or equal to $38^{\circ} \mathrm{C}\left(101^{\circ} \mathrm{F}\right)$ for 1 hour with an absolute neutrophil count $(\mathrm{ANC})<500$ cells $/ \mu \mathrm{L}$. If neutrophils drop rapidly, this is considered a higher risk of infection and death. More than two-thirds of fever episodes in neutropenic patients are likely caused by infection. Damage to the host by chemotherapy and radiation and the potential access of the organism through the central venous pathway can lead to microbial invasion. Bacteremia occurs in 10\%$25 \%$ of all patients, with most episodes occurring in prolonged or severe levels of neutropenia (ANC <100 cells $/ \mu \mathrm{L}$ ). Patients with bacterial infections cannot be distinguished precisely from uninfected patients at first. Because the development of infection in neutropenic patients can be very rapid and there is a possibility that there are no typical infectious symptoms such as fever and swelling. Symptoms of respiratory disorders such as cough, runny nose, sore throat, shortness of breath, and pleuritic chest pain can also be found along with fever [2].

Febrile neutropenic patients have a low risk of complications if they have a good performance status and several comorbid medical conditions balanced by adequate liver function and kidney function, and the duration of neutropenia is estimated to be less than seven days. Febrile neutropenic patients have a high risk of complications if they experience severe neutropenia characterized by ANC $<100$ cells $/ \mu \mathrm{L}$ after chemotherapy, and the duration of neutropenia is estimated to last longer than seven days. In addition, high-risk patients may have clinically relevant comorbidities such as hypotension, pneumonia, renal or liver insufficiency, or neurological changes [7].

To note, the examinations that must be done on febrile neutropenic patients include physical examinations that include skin lesions, mucous membranes, intravenous catheter locations, perirectal areas [8], and laboratory examinations that include complete blood counts and blood cultures [9].

Based on the description above, this study is needed to determine the clinical and hematological profile of pediatric patients with febrile neutropenia who suffered from malignancy at the Division of HematologyOncology, Department of Pediatrics, Dr. Soetomo General Academic Hospital, Surabaya.

\section{Methods}

This research method is a descriptive retrospective study using secondary data from the medical record of pediatric patients with febrile neutropenia who suffered from malignancy at the Pediatric HematologyOncology Inpatient Installation, Department of Pediatrics, Dr. Soetomo General Academic Hospital in 
January 2018-December 2019. This study used a non-random sampling technique with a total of 30 pediatric patients with febrile neutropenia who suffered from malignancy. The characteristics of the samples taken in this study are the underlying clinical diagnosis of neutropenic fever, clinical symptoms, clinical signs, and the results of hematological examinations. This research has been approved by the Health Research Ethics Committee of Dr. Soetomo General Academic Hospital (0144/LOE/301.4.2/X/2020).

\section{Results}

The data collection in this study was conducted with medical records in pediatric patients with febrile neutropenia who suffered from malignancy at Dr. Soetomo General Academic Hospital from January 2018 to December 2019. Thirty pediatric patients with febrile neutropenia suffered from malignancy based on the data. The characteristics of the samples taken in this study are the underlying clinical diagnosis of febrile neutropenia, clinical symptoms, clinical signs, and the results of hematological examinations.

Table 1. Underlying Clinical Diagnosis in Pediatric Patients with Febrile Neutropenia who Suffered from Malignancy at Dr. Soetomo General Academic Hospital in 2018-2019

\begin{tabular}{lll}
\hline Clinical Diagnosis & Frequency (n) & Percentage (\%) \\
\hline Acute Lymphoblastic Leukemia & 22 & 73 \\
Extraocular Retinoblastoma & 1 & 3 \\
Acute Myelocytic Leukemia & 1 & 3 \\
Chronic Myelocytic Leukemia & 1 & 3 \\
Suspected Acute Leukemia & 1 & 3 \\
Burkitt's Lymphoma & 1 & 3 \\
Neuroblastoma & 1 & 3 \\
Germ Cell Tumor & 1 & 3 \\
Osteosarcoma & 1 & 3 \\
\hline
\end{tabular}

Table 1 shows the underlying clinical diagnosis, both primary and secondary diagnosis in pediatric patients with febrile neutropenia who suffered from malignancy. Based on Table 1, the most common clinical diagnosis experienced by pediatric patients with febrile neutropenia who suffered from malignancy is acute lymphoblastic leukemia, as many as 22 patients $(73 \%)$.

Table 2. Clinical Symptoms in Pediatric Patients with Febrile Neutropenia who Suffered from Malignancy at Dr. Soetomo General Academic Hospital in 2018-2019

\begin{tabular}{lll}
\hline Clinical Symptoms & Frequency (n) & Percentage (\%) \\
\hline Fever & 30 & 100 \\
Shortness of Breath & 5 & 17 \\
Cough & 2 & 7 \\
Pain in The Perirectal Area & 1 & 3 \\
Swelling or Pain in The Catheter Area & 0 & 0 \\
\hline
\end{tabular}

Table 2 shows clinical symptoms in pediatric patients with febrile neutropenia who suffered from malignancy from febrile neutropenia. Based on Table 2, the most common clinical symptoms experienced by 
pediatric patients with febrile neutropenia who suffered from malignancy are fever, as many as 30 patients $(100 \%)$.

Table 3. Clinical Signs in Pediatric Patients with Febrile Neutropenia who Suffered from Malignancy at Dr. Soetomo General Academic Hospital in 2018-2019

\begin{tabular}{lll}
\hline Clinical Signs & Frequency $(\mathrm{n})$ & Percentage $(\%)$ \\
\hline Anemia & 20 & 67 \\
Hepatomegaly & 2 & 7 \\
Pneumonia Infection & 2 & 7 \\
Splenomegaly & 1 & 3 \\
\hline
\end{tabular}

Table 3 shows clinical signs in pediatric patients with febrile neutropenia who suffered from malignancy from febrile neutropenia. Based on Table 3, the most common clinical sign experienced by pediatric patients with febrile neutropenia who suffered from malignancy is anemia, as many as 20 patients (67\%).

Table 4. Results of Hematology Examination in Pediatric Patients with Febrile Neutropenia who Suffered from Malignancy at Dr. Soetomo General Academic Hospital in 2018-2019

\begin{tabular}{lll}
\hline Hematology Examination & Frequency $(\mathrm{n})$ & Percentage $(\%)$ \\
\hline Neutrophils $(<500$ cells $/ \mu \mathrm{L})$ & 30 & 100 \\
Platelets $(<150.000$ cells $/ \mu \mathrm{L})$ & 22 & 73 \\
Leukocytes $(<4000$ cells $/ \mu \mathrm{L})$ & 21 & 70 \\
Hemoglobin $(<11-13$ grams $/ \mathrm{dL})$ & 20 & 67 \\
\hline
\end{tabular}

Table 4 shows the results of hematology examinations in pediatric patients with febrile neutropenia who suffered from malignancy. Based on Table 4, the most common hematology examination results found in pediatric patients with febrile neutropenia who suffered from malignancy from febrile neutropenia are neutropenia, as many as 30 patients $(100 \%)$, followed by thrombocytopenia as many as 22 patients (73\%), leukopenia as many as 21 patients $(70 \%)$, and anemia as many as 20 patients $(67 \%)$.

Table 5. Range and Average Amount of Neutrophils, Platelets, and Hemoglobin Levels in Pediatric Patients with Febrile Neutropenia who Suffered from Malignancy at Dr. Soetomo General Academic Hospital in 2018-2019

\begin{tabular}{lll}
\hline Hematology Examination & Range & Average \\
\hline Neutrophils $(<500$ cells $/ \mu \mathrm{L})$ & $0-440$ & 179.23 \\
Platelets $(<150.000$ cells $/ \mu \mathrm{L})$ & $1230-141000$ & 34716.81 \\
Leukocytes $(<4000$ cells $/ \mu \mathrm{L})$ & $210-2110$ & 1129.76 \\
Hemoglobin $(<11-13$ grams $/ \mathrm{dL})$ & $1.8-10.5$ & 8.95 \\
\hline
\end{tabular}

Table 5 explains in detail that the average number of neutrophils is 179.23 cells $/ \mu \mathrm{L}$, the average platelet count is 34716.81 cells $/ \mu \mathrm{L}$, the average number of leukocytes is 1129.76 cells $/ \mu \mathrm{L}$, and the average hemoglobin level is 8.95 grams/dL. 


\section{Discussion}

This study explains the clinical and hematological profile in pediatric patients with febrile neutropenia who suffered from malignancy at Dr. Soetomo General Academic Hospital Surabaya. Based on data obtained from the medical record of Dr. Soetomo General Academic Hospital Surabaya in the period January 2018December 2019, it was found that the clinical diagnosis underlying the occurrence of febrile neutropenia in pediatric patients with malignancy was acute lymphoblastic leukemia, as many as 22 patients (73\%) out of a total of 30 patients, as stated in Table 1. There are similarities between the current research and previous research conducted by Amonkar Priyanka (2018) and Suha Al Omar et al (2013). In a study conducted by Amonkar Priyanka at Goa Medical College's Department of Pediatrics in 2018, it was obtained that pediatric patients who had febrile neutropenia and had acute lymphoblastic leukemia, which was as many as 41 patients (82\%) [10]. Suha Al Omar et al (2013), by taking data from King Hussein Cancer Center (KHCC) in Jordan for six months, also obtained child patients who have febrile neutropenia and have acute lymphoblastic leukemia, as many as 60 patients (55\%) [11]. Most standard chemotherapy regimens used in treating childhood cancer, both in hematological malignancies and solid tumors, cause periods of myelosuppression with neutropenia often lasting more than seven days [12].

Based on this study, the most common symptoms and clinical signs experienced by pediatric patients with febrile neutropenia who suffered from malignancy are fever, as many as 30 patients (100\%) out of 30 patients as stated in Table 2. While other clinical symptoms, such as shortness of breath, cough, and pain in the perirectal area, are only complained of as many as 5 patients (17\%), 2 patients (7\%), and 1 patient (3\%). A study conducted by Rajan Kapoor et al at Army Hospital, New Delhi in 2018, found child patients with neutropenia fever who had a fever, as many as 50 patients (100\%). It is followed by respiratory symptoms such as cough, cold, sore throat, shortness of breath, and sometimes pleuritic chest pain, as many as 16 patients (32\%) [2]. From the results of current research data with previous research conducted by Rajan Kapoor (2018), there are similarities, namely the symptoms and clinical signs most suffered by pediatric patients with febrile neutropenia who suffered from malignancy is fever.

In this study, the most common clinical sign experienced by pediatric patients with febrile neutropenia who suffered from malignancy was anemia, as many as 20 patients $(67 \%)$ out of a total of 30 patients, as stated in Table 3. Specifically, anemia is classified into 3, namely mild anemia as many as 7 patients (35\%), moderate anemia as many as 12 patients $(60 \%)$, and severe anemia as many as 1 patient $(5 \%)$. A study conducted by Rajan Kapoor et al at Army Hospital, New Delhi in 2018, found pediatric patients with malignancy who had febrile neutropenia and severe anemia as much as 10\% [2]. When viewed from previous and current studies, cases of severe anemia are rare. Hemoglobin with a level of $\geq 9$ grams/dL is more commonly found and commonly associated with the risk of adverse drug events (ADE) [13].

Based on this study, the most common hematology examination results found in pediatric patients with febrile neutropenia who suffered from malignancy are neutropenia (ANC $<500$ cells $/ \mu \mathrm{L}$ ), as many as 30 patients (100\%), thrombocytopenia as many as 22 patients (73\%), leukopenia as many as 21 patients $(70 \%)$, and anemia as many as 20 patients (67\%), out of a total of 30 patients, as stated in Table 4 and 5 . In a study conducted by Soner Sertan Kara et al at the hematology-oncology clinic of the Faculty of Medicine of Gazi University and the Pediatric Oncology Hematology Research and Training Hospital in Ankara, Turkey, in 2019, children with febrile neutropenia experienced changes in the number of absolute neutrophils, as many as 150 patients (100\%) with different neutropenia severity variations. Details of the results of hematology examination in the study are the median value of absolute neutrophil count (ANC) is 40 cells $/ \mu \mathrm{L}$, an average hemoglobin level is 8.8 grams/dL, and the median value of platelet count is 38,550 cells $/ \mu \mathrm{L}$ [14]. Current and previous studies show that neutropenia results from hematological examinations are widely found in pediatric patients with febrile neutropenia who suffer from malignancy. 


\section{Conclusion}

From this study, it is known that the most common clinical diagnosis was acute lymphoblastic leukemia. The most common clinical symptom experienced by patients was fever. The most common clinical sign experienced by patients was anemia. The most common hematological examination results were neutropenia with absolute neutrophil count $<500$ cells $/ \mu \mathrm{L}$. Further research is needed to determine febrile neutropenia's clinical and hematological profile based on a complete blood test, blood culture examination, and treatment obtained by the patient while being treated at the Pediatric Hematology-Oncology Inpatient Installation, Department of Pediatrics, Dr. Soetomo General Academic Hospital.

\section{Acknowledgement}

The authors would like to thank the Director of Dr. Soetomo General Academic Hospital and Department of Pediatrics, Dr. Soetomo General Academic Hospital for supporting this research.

\section{References}

[1] Nursyirwan, S. R. \& Windiastuti, E., 2017. Kejadian Demam Neutropenia pada Anak dengan Keganasan. Sari Pediatri, 19(4), pp. 220-225. DOI: http://dx.doi.org/10.14238/sp19.4.2017.220-5.

[2] Kapoor, R., Simalti, A. K., Roy, S. \& Agarwal, P., 2018. Clinicohematological Profile of Febrile Neutropenia in Childhood Acute Leukemia and Utility of Serum Procalcitonin Levels in Neutropenic Patients. Indian J Crit Care Med, 22(5), pp. 336-339. DOI: 10.4103/ijccm.IJCCM_516_17.

[3] Weycker, D. et al., 2015. Risk and Consequences of Chemotherapy-Induced Febrile Neutropenia in Patients with Metastatic Solid Tumors. Journal of Oncology Practice, pp. 47-54. DOI: 10.1200/JOP.2014.001492.

[4] Teranishi, H. et al., 2017. Clinical Efficacy of Cycling Empirical Antibiotic Therapy for Febrile Neutropenia in Pediatric Cancer Patients. Journal of Infection and Chemotherapy, pp. 463-467. DOI: 10.1016/j.jiac.2017.03.020.

[5] Lekshminarayanan, A. et al., 2018. National Trends in Hospitalization for Fever and Neutropenia in Children with Cancer, 2007-2014. The Journal of Pediatrics, pp. 232-237. DOI: 10.1016/j.jpeds.2018.06.056.

[6] Lucas, A. J., Olin, J. L. \& Coleman, M. D., 2018. Management and Preventive Measures for Febrile Neutropenia. Pharmacy and Therapeutics, 43(4), pp. 228-232.

[7] The American Journal of Managed Care, 2017. Guidelines in the Management of Febrile Neutropenia for Clinical Practice. The American Journal of Managed Care.

[8] Longo, D. L., 2010. Harrison's Hematology and Oncology. New York: McGraw-Hill Medical.

[9] Segel, G. B. \& Halterman, J. S., 2008. Neutropenia in Pediatric Practice. Journal of The American Academy of Pediatrics, 29(1), pp. 12-24. DOI: 10.1542/pir.29-1-12.

[10] Priyanka, A. \& Vaishali, J., 2018. Clinical Profile of Febrile Neutropenia in Children with Cancer. Indian Journal of Applied Research, 8(8), pp. 53-54. DOI: 10.36106/ijar.

[11] Omar, S. A., Nazer, L. \& Alkayed, K., 2013. A Prospective Study of Febrile Neutropenia in Pediatric Cancer Patients in Jordan. Journal of Pediatric Hematology/Oncology, 35(8), pp. 614-617. DOI: 10.1097/MPH.0b013e31829f3480.

[12] Davis, K. \& Wilson, S., 2020. Febrile Neutropenia in Paediatric Oncology. Paediatric Child Health, 30(3), pp. 93-97. DOI: https://doi.org/10.1016/j.paed.2019.12.002. 
[13] Ammann, R. A., Niggli, F. K., Leibundgut, K., Teuffel, O., \& Bodmer, N. (2014). Exploring the association of hemoglobin level and adverse events in children with cancer presenting with fever in neutropenia. PloS one, 9(7), e101696. DOI: 10.1371/journal.pone.0101696.

[14] Kara, S. S. et al., 2019. Risk Factors for Bacteremia in Children with Febrile Neutropenia. Turkish Journal of Medical Sciences, 49(4), pp. 1198-1205. DOI: 10.3906/sag-1901-90. 